Cell Research (1999), 9, 291-303

\title{
Leukolysin/MMP25/MT6-MMP: a novel matrix metalloproteinase specifically expressed in the leukocyte lineage
}

\author{
Pei Duanqing \\ Department of Pharmacology, 6-120 Jackson Hall, 321 Church \\ St. S.E., University of Minnesota, Minneapolis, MN 55455, USA \\ Tel: 612-626-1468; Fax: 612-625-8408; \\ E-mail:peixx003@tc.umn.edu
}

\begin{abstract}
A novel matrix metalloproteinase (MMP) was identified from leukocytes and found to be specifically expressed by peripheral blood leukocytes among 29 different tissues examined. Named leukolysin, it encodes for 562 residues with a conserved MMP structure, i.e, pre-, pro-, catalytic-, hinge- and hemopexinlike domains, but also a RXK/RR motif, known for its role in MMP zymogen activation, and a C-terminal hydrophobic segment. Overall, leukolysin displays the strongest homology to the newly identified MT-MMP subgroup with $45 \%$ and $39 \%$ identities to MT4- and MT1-MMPs vs $30 \%$ and $31.5 \%$ to MMP1 and 3 respectively. Unlike MT4-MMP whose proteolytic activity remains undefined, a C-terminally truncated leukolysin is expressed as a strong gelatinolytic species at $28 \mathrm{kDa}$ which is derived from a cell-associated $34 \mathrm{kDa}$ proenzyme, presumably by furin or proprotein convertase mediated removal of the propeptide $(\sim 6 \mathrm{kDa})$. By green fluorescent protein (GFP) tagging, the intracellular proenzyme is localized to granules throughout the cell, suggesting that activation occur immediately prior to secretion. Taken together, leukolysin may be part of the proteolytic arsenal deployed by leukocytes during inflammatory responses.
\end{abstract}

The nucleotide sequence reported in this paper has been submitted to the Gen Bank TM/EMBL Data Bank with accession number AF185270 
Molecular cloning of a novel MMP: MMP:25

Key words: $M T 6-M M P, M M P 25$, leukolysin, MMP, Matrix Remodeling, Leukocytes.

\section{INTRODUCTION}

Members of the matrix metalloproteinases (MMP) have been consistently implicated in the destruction of extracellular matrix (ECM) under many pathophysiological conditions from angiogenesis to arthritis[6,7]. To keep these powerful proteinases in latent form, most MMPs rely on a conserved cysteine in the prodomains to interact with the catalytic zinc according to the cysteine-switch latency model ADDIN ENRfu [8]. The only exception is the newly identified CA-MMP which lacks a cysteine-switch in the prodomain[9]. At the carboxyl-termini, a hemopexin-like domain is found in all MMPs except CA-MMP and matrilysin[9-11]. Recently, two additional motifs have been recognized within the MMP family: 1) a RXK/RR motif for furin or proprotein convertase (PC) mediated zymogen activation[12-14]; and 2) a hydrophobic segment at the carboxyl termini of MMP14-17 consistent with that of a transmembrane domain[15-18]. Based on these functional domains, a diverse family of MMPs must have been evolved to accomplish the tasks of ECM remodeling necessary for the growth and development of multicellular organisms such as human. In this report, a novel MMP named leukolysin was identified in leukocytes. Known for their roles in inflammatory response[4], white cells may deploy leukolysin when exiting the blood stream to reach the inflammatory sites and/or mediating the destruction of invading pathogens and infected host tissues.

\section{MATERIALS AND METHODS}

\section{Cell lines and reagents}

Mammalian cells including MDCK cells and COS 7 are cultured as described[19], [20]. Reagents associated with recombinant DNA techniques were purchased from Promega (Madison, WI). The RT-PCR/RACE kit is from Roche Diagnostics (Indianapolis, IN). Oligonucleotide primers were made by either the University of Minnesota microchemical core facility or IDT (Coralville, IA). FLAG peptide, anti-FLAG M2 monoclonal antibody and agarose beads were purchased from Sigma (St. Louis, MO).

\section{Molecular cloning and sequence analysis of human leukolysin}

Initially, EST clones (AI150017 and AA830519, both from IMAGE consortium[21]) were identified through a BLAST search to encode a putative MMP showing 60\% identity to Leu235Gly298 of MT4-MMP[18], [22]. Based on these sequences, subsequent RT-PCR and RACE reactions using RNA isolated from human leukocytes were performed to isolated a composite $2.6 \mathrm{~kb}$ cDNA which encodes an open reading frame. Sequencing and sequence analysis was performed 
as described previously[20].

\section{Tissue distribution of leukolysin}

Human cDNA panels were purchased from Clontech (Palo Alto, CA) and amplified for the expression of leukolysin with two primers (TAC GCT CTG AGC GGC AGC and CCC ATA GAG TTG CTG CAG) at the following conditions: 2 min at $94^{\circ} \mathrm{C}$ for denaturation, 35 cycles of 10 sec at $94^{\circ} \mathrm{C}$ for denaturation, $30 \mathrm{sec}$ at $52^{\circ} \mathrm{C}$ for annealing and $30 \mathrm{sec}$ at $72^{\circ} \mathrm{C}$ for extension, followed by $10 \mathrm{~min}$ extension at $72^{\circ} \mathrm{C}$. The house-keeping gene GAPDH was used as internal references as described previously[9].

\section{Expression vectors for leukolysin1-280, leukolysin1-280-GFP and generation of their stable transfectants}

The first 280 residues of leukolysin was isolated by high-fidelity PCR with pfu polymerase (15 cycles) and inserted into two vectors: pCR3.1-FLAG and pCR3.1-GFP-FLAG (Pei, etal unpublished). The 3 ' end of the insert was designed such that it is inserted in-frame with FLAG and GFP-FLAG respectively. The resulting vectors were sequenced and confirmed to be errorfree and in-frame as designed. The resulting expression vectors were transfected into MDCK cells and stable clones were obtained by G418 selection[19]. More than 20 clones were isolated for each transfection. For screening, serum-free conditioned media from each clone were analyzed by gelatin-zymography and Western blot using M2 antibody[9]. For analysis of cell associated recombinant protein, cells were lysed in RIPA buffer (50 mM Tris, pH 7.5, $150 \mathrm{mM} \mathrm{NaCl}, 0.25 \%$ sodium deoxycholate, $0.1 \%$ Nonidet P-40, $10 \mu \mathrm{M}$ leupeptin, $0.1 \mu \mathrm{M}$ 5-APMSF, $1 \mathrm{mM}$ aprotinin). Cleared of any debris by microcentrifugation, the lysates were fractionated on SDS-PAGE and Western blotted with M2 antibody as described above.

\section{Confocal microscopy}

Cells were washed twice in PBS, fixed in 4\% paraformaldehyde (30 min) and examined on a BioRad MRC confocal system maintained at the Biological Imaging and Processing Laboratory of the University of Minnesota.

\section{RESULTS}

\section{Identification of a novel MMP named leukolysin/MMP25/MT6-MMP}

Searching the EST database for novel MMP-like genes have resulted in the identification of two latest additions to the MMP family: CA-MMP/MMP23 and MT5-MMP/MMP24 [9],[11], [20],[23]. Continuing this effective strategy, a putative MMP gene was identified (see materials and methods), which shares considerable homology to MT4-MMP, initially reported by Puente et al[18] and recently completed by Kajita and colleagues[22] As shown in Fig 1, this novel MMP possesses a MMP domain structure with 1) a cysteineswitch PRCXXPD in the prodomain, 2) a HEXGHXXXXXH zinc binding motif in the catalytic domain and 3) a hemopexin-like domain at the C-terminal portion. Interestingly, with a RXR/KR motif between its pro- and catalytic domains, this novel MMP apparently joins a subfamily of MMPs with a well-defined mechanism of zymogen activation mediated by members of the proprotein convertases such as furin in the trans-Golgi network 
Molecular cloning of a novel MMP: MMP:25

$[12],[14],[24]$. At the C-terminus, a hydrophobic segment is present as a potential transmembrane domain (Fig 1b). Given its origin of isolation and tissue specific expression in leukocytes (See Fig 2), this novel gene is tentatively named leukolysin in analogy to stromelysins. Following the MMP serial designation, it isassigned MMP25 (personal communications, J.F. Woessner, Jr., University of Miami, FL). On the other hand, due to its close relationship to MT4-MMP and the other MT-MMPs, MT6-MMP is also proposed for this gene.

The relationship between leukolysin and other MMPs

To further analyze the relatedness between leukolysin and known MMPs, a multiple sequence alignment was constructed and represented in Fig 1c. Based on the alignment

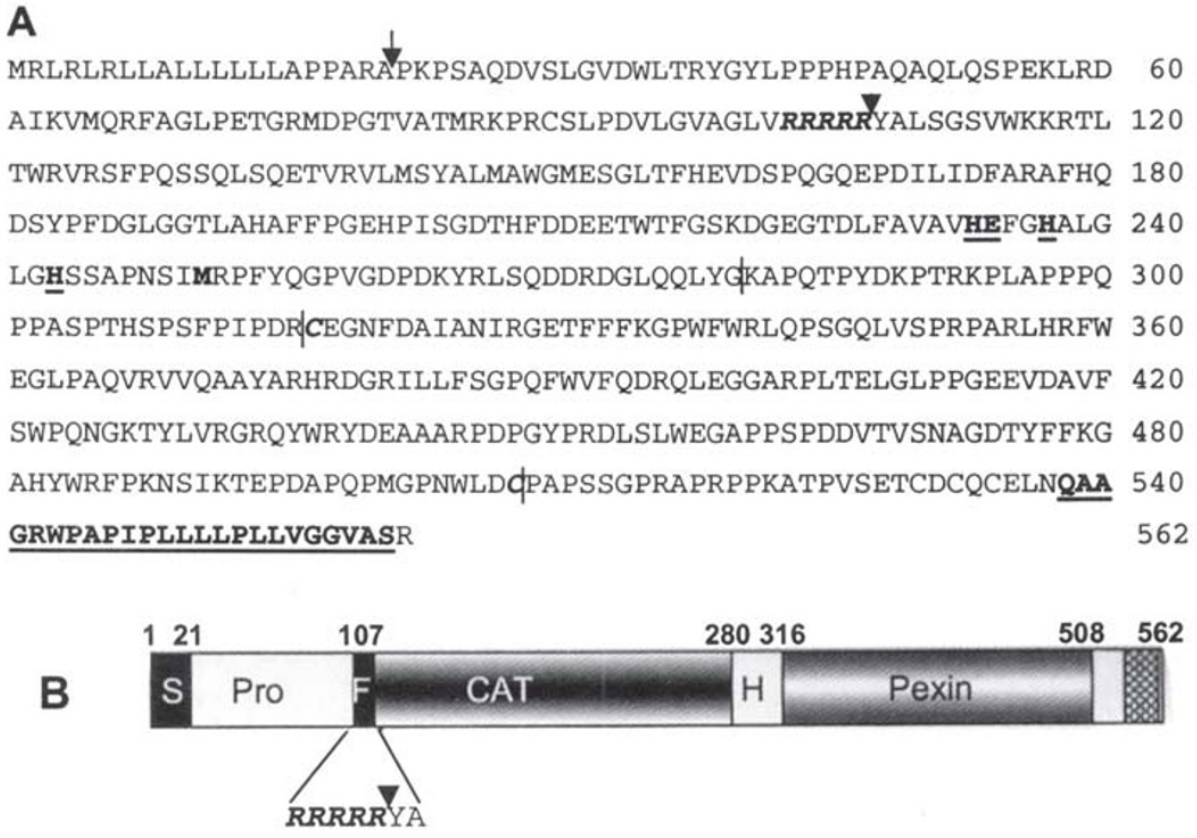

Fig 1.

Leukolysin: primary sequence, domain structure and alignments with MT-MMPs.

A, The amino acid sequence is shown with markings for the signal peptide cleavage site (downward arrow), putative zymogen activation cleavage site by furin (bold and italicized letters with a downward arrowhead), the conserved catalytic zinc binding domain (bold and underlined), M turn, the hemopexin-like domain bound by a pair of cysteines (bold and italicized), and the hydrophobic segment at the C-terminus (bold and underlined). B, A schematic representation of domain structure for leukolysin. The numbers on the top indicate the positions of the amino acid residues bordering various domains. S: signal peptide; Pro: prodomain; F: furin recognition motif; CAT: catalytic; H: hinge; Pexin: hemopexin-like. 
MT3

MT5

MT2

MT1

MT4

MMP25

MT3

MT5

MT2

MT1

MT4

MMP25

MT3

MT5

MT2

MT1

MT4

MMP25

MT3

MT5

MT2

MT1

MT4

MMP25

MT3

MT5

MT2

MT1

MT4

MMP25

MT3

MT5

MT2

MT1

MT4

MMP25

MT3

MT5

MT2

MT1

MT4

MMP25

MT 3

MT5

MT2

MT1

MT4

MMP2 5

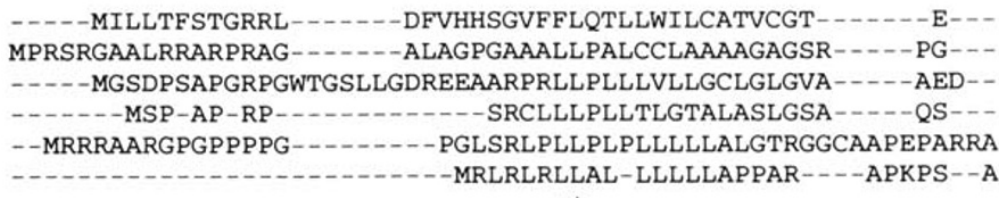

-QYFNVEVWLQKYGYLPPTDPRMSVLRSAETMQSALAAMQQFYGINMTGKVDRNTIDWMK -RPFAGQNWLKSYGYLLPYESRASALHSGKALQSAVSTMQQFYGIPVTGVLDQTTIEWMK - AEVHAENWLRLYGYLPQPSRHMSTMRSAQILASALAEMQRFYGIPVTGVLDEETKEWMK -SSFSPEAWLQQYGYLPPGDLRTHTQRSPQSLSAAIAAMQKFYGLQVTGKADADTMKAMR EDLSLGVEWLSRFGYLPPADPTTGQLQTQEELSKAITAMQQFGGLEATGI LDEATLALMK QDVSLGVDWLTRYGYLPPPHPAQAQLQSPEKLRDAIKVMQRFAGLPETGRMDPGTVATMR

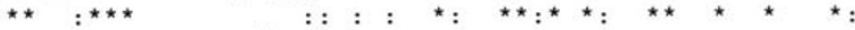
Furin cleavage site $\downarrow$

KPRCGVPDQTRGSSKFHY--RRKRYALTGQKWQHKHITYSIKNVT- - PKVGDPETRKAIR KPRCGVPDHPHLS----RRRRNKRYALTGQKWRQKHITYSIHNYT- - PKVGELDTRKAIR RPRCGVPDQFGVRVKANLRRRRKRYALTGRKWNNHHLTFSIQNYT- - EKLGWYHSMEAVR RPRCGVPDKFGAEIKANN--RRKRYAIQGLKWQHNEITFCIQNYT--PKVGEYATYEAIR TPRCSLPDLP-VLTQA---RRRRQAPAPTKWNKRNLSWRVRTFPRDSPLGHDTVRALMY KPRCSLPDVLGVAGLV--RRRRRYALSGSVWKKRTLTWRVRSFPQSSQLSQETVRVLMS

$$
{ }^{\star \star \star} .::^{\star \star} \quad{ }^{\star} \text {. }{ }^{\star} \text {. : . : : : : : : : . . . } \quad \text { : }
$$

RAFDVWQNVTPLTFEEVPYSELENGK-RDVDITI IFASGFHGDSSPFDGEGGFLAHAYFP QAFDVWQKVTPLTFEEVPYHEIKSDR-KEADIMIFFASGFHGDSSPFDGEGGFLAHAYFP RAFRVWEQATPLVFQEVPYEDIRLRRQKEADIMVLFASGFHGDSSPFDGTGGFLAHAYFP KAFRVWESATPLRFREVPYAYIREGHEKQADIMIFFAEGFHGDSTPFDGEGGFLAHAYFP YALKVWSDIAPLNFHEV - - - -AGS- - TADIQIDFSKADHNDGY PFDARR-HRAHAFFP YALMAWGMESGLTFHEVD---SPQGQ--EPDILIDFARAFHQDSYPFDGLGGTLAHAFFP

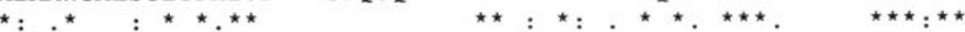
Active site

GPG - IGGDTHFDSDEPWTLGNPNHDGNDLFLVAVHELGHALGLEHSNDPTA IMAPFYQ- GPG-IGGDTHFDSDEPWTLGNANHDGNDLFLVAVHELGHALGLEHSNDPSA IMAPFYQ- GPG-LGGDTHFDADEPWTFSSTDLHGNNLFLVAVHELGHALGLEHSSNPNA IMAPFYQ- GPN-IGGDTHFDSAEPWTVRNEDLNGNDIFLVAVHELGHALGLEHSSDPSA IMAPFYQ- GHHHTAGYTHFNDDEAWTFRSSDAHGMDLFAVAVHEFGHAIGLSHVAAAHSIMRPYYQGP GEHPISGDTHFDDEETWTFGSKDGEGTDLFAVAVHEFGHALGLGHSSAPNS IMRPFYQGP

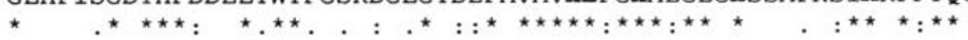

YMETDNFKLPNDDLQGIQKIYGPPDKI PPPTRPLPTVPPHRSIPPADPRKNDR-PKPPRP YMETHNF KLPQDDLQGIQKIYGPPAEPSGATRPLPTLPVRRIHSPSE-KKHERHPRPPRR WKDVDNFKL PEDDLRGIQQLYGTPDGQPQPTQPLPTVTPRRPGRPDH- - - RPPRPPQP WMDTENFVLPDDDRRGIQQLYGGESG - - - - FPTKMPPQP-RTTS- - - - RPSVPDVGDPLRYGLPYEDKVRVWQLYGVRESVSPTAQ- - PEEP PLLP - - - - - - EP - PDNR VGDPDKYRLSQDDRDGLQQLYG KAPQTPYDK- - PTRKPLAP - - - - - - - PPQPPAS

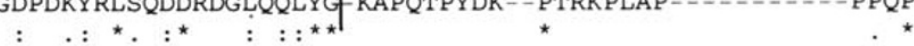

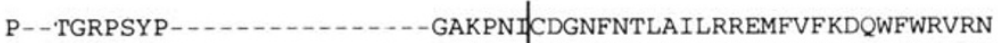
P--WGRPSTP _.......... GAKPNICDGNFNTVALFRGEMFVFKDRWFWRLRN PPPGGKPERPPKPGPPVQPRATERPDQYGPNICDGDFDTVAMLRGEMFVFKGRWFWRVRH _..-KPKNP_... S--SAPPRKD - . P--THSPSFP

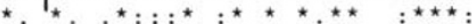

NR-VMDGYPMOITYFWRGLPP---SIDAVYENS - DGNFVFFKGNKYWVFKDTTLQPGYPH NR-VQEGYPMQIEQFWKGLPA---RIDAAYERA-DGRFVFFKGDKYWVFKEVTVEPGYPH NR-VLDNY PMPIGHFWRGLPG---DISAAYERQ-DGRFVFFKGDRYWLFREANLEPGY PQ NQ-VMDGYPMPIGQFWRGLPA---SINTAYERK-DGKFVFFKGDKHWVFDEASLEPGYPK DRHLVSLQPAQMHRFWRGLPLHLDSVDAVYERTSDHKIVFFKGDRYWVFKDNNVEEGY PR SGQLVSPRPARLHRFWEGL PAOVRVVOAAYARHRDGRI LLFSGPQFWVFODRQLEGG-AR

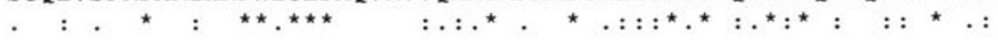


Molecular cloning of a novel MMP: MMP:25

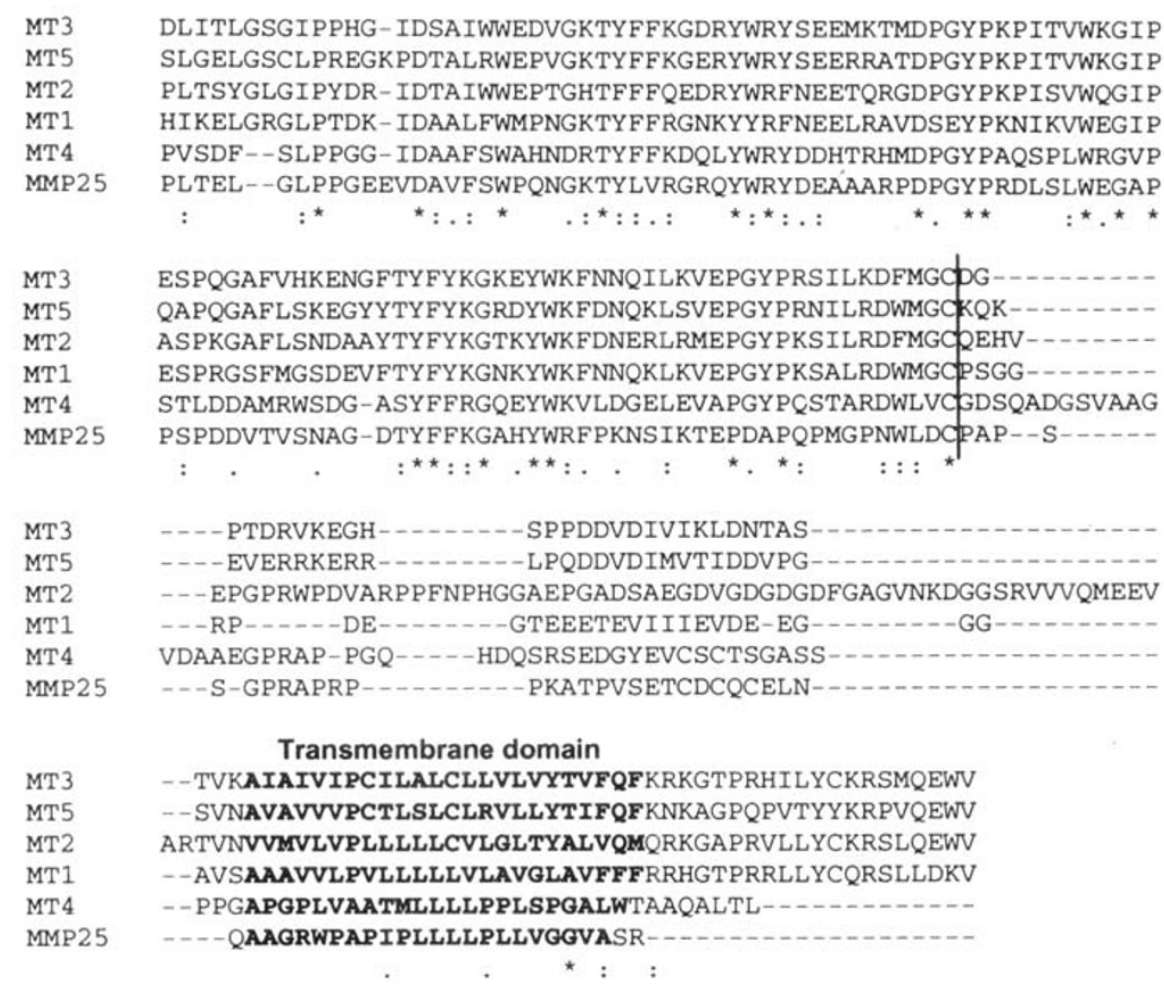

Fig 1.

C, Sequence alignment between leukolysin (shown as MMP25) and the MT-MMP subgroup. The names of each gene are shown on the left column: MT1-5 = MT1-5MMPs. The cysteine-switches, the catalytic and transmembrane domains are shown in bold letters. The furin cleavage sites are boxed and indicated by a downward arrow. The end of the catalytic domains and the beginning and end of the hemopexin domains are marked by vertical lines. * indicates identity, : strong homology and. weak homology.

score, leukolysin shows the highest homology to MT4-MMP (45\% identity overall), a putative MMP with unknown enzymic and biological function. In addition, leukolysin has the strongest homology to the rest of the MT-MMP subgroup among all MMPs identified so far. In fact, the catalytic domain of leukolysin, the most conserved part of the MMP family, displays 56\% amino acid sequence identity to MT4-MMP, compared to 46$48 \%$ for MT1, 2, 3, 5-MMPs (Fig 1c). Interestingly, the C-termini are the most divergent and generally fall into two groups. The first group, including MT1, 2, 3, and 5-MMPs, 
has a traditional transmembrane domain of 24 residues followed by a cytosolic domain with a conserved Valine at their termini (Fig 2a). The second group, made of MT4-MMP and leukolysin, has no visible cytosolic domains after the hydrophobic domains (Fig 1c).

\section{Tissue specific expression of leukolysin}

To investigate the distribution of leukolysin, a panel of cDNAs prepared from 26 tissues and organs were screened for leukolysin expression by PCR. As shown in Fig 2, peripheral leukocytes are the main source of leukolysin. Bone marrow and fetal kidney also express minute amounts of leukolysin, probably reflecting residual blood leukocytes in those organs at the time of sample collections.

\section{Expression of leukolysin as a soluble enzyme}

As shown in Fig 1, leukolysin possesses a hydrophobic segment which could anchor the protein on cell surface, thus, making purification of the proteinase difficult. Indeed, fulllength as well as the transmembrane truncated leukolysins are expressed as cell associated forms (Pei, unpublished data). To circumvent potential problem, a vector was constructed to express leukolysin $\sin _{1-28}$ tagged with FLAG epitope, without the pexin- and putative transmembrane domains (Fig 3a). Since leukolysin contains a furin site between its pro- and catalytic-domains, it is expected that leukolysin ${ }_{1-280}$ will be expressed in latent form in the ER, transported to the Golgi where it is processed into active forms as described for MMP11 and MMP14[12],[13],[25]. The expression vector, pCR3. 1MMP25cat, was transfected into MDCK cells and stable clones were isolated and screened by zymography. As shown in Fig $3 \mathrm{~b}$, a $28 \mathrm{kDa}$ gelatinolytic species was detected in the conditioned media of several transfectants (lanes 3,4 and 6). This finding is in sharp contrast to the negative results obtained when similar MT4-MMP was expressed under identical conditions (Trui and Pei, unpublished data). One of the clones with high yields, 17-13 (Fig 3b, lane 3), was chosen for further characterization.

\section{Soluble leukolysin is secreted as a processed and fully active enzyme}

With a RRRR motif, leukolysin is expected to be activated by furin or related PCs intracellularly prior to secretion. Thus, the conditioned media and cell lysates were analyzed by Western blot to establish the relationship between extracellular and intracellular leukolysins. As shown in Fig 3c, the conditioned media from 17-13 contains a major immuno-reactive species at $\sim 28 \mathrm{kDa}$ and a minor one at $\sim 34 \mathrm{kDa}$ (Fig 3c, lanes 3 ), potentially corresponding to the active and pro- forms of leukolysin. In contrast, more than $97 \%$ of intracellular leukolysin is in the $34 \mathrm{kDa}$ form, compared to $\sim 3 \%$ for the 28 $\mathrm{kDa}$ active forms (Fig 3c, lane 5), thus validating a precusor/product relationship for the 34 and $28 \mathrm{kDa}$ species. The loss of $\sim 6 \mathrm{kDa}$ is presumably the result of prodomain processing at the RRRR signal by furin or its related PCs[12]. 
Molecular cloning of a novel MMP: MMP:25

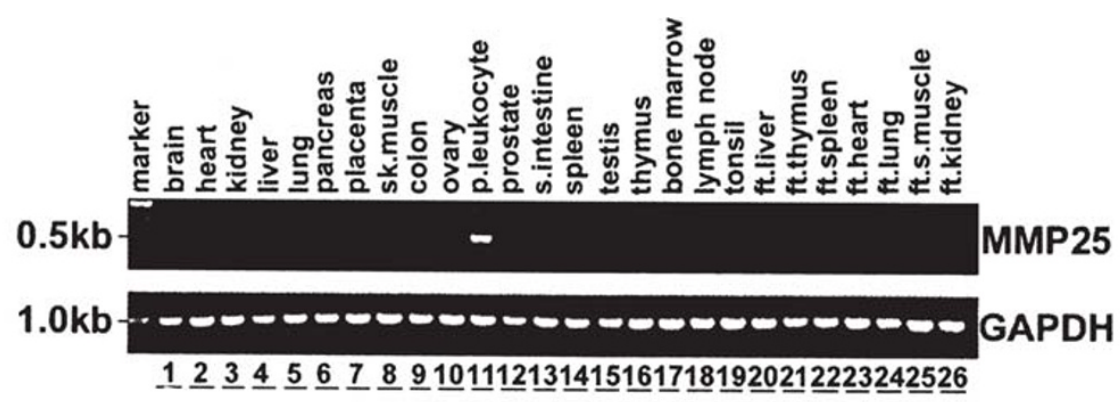

Fig 2.

Leukolysin is expressed specifically by neutrophils.

Reverse transcribed cDNA (0.5 ng aliquot each) from human multiple tissue panels (Clontech, CA) including 26 different tissues and cell types as indicated at the top (lanes 1-26) were amplified to give rise to $\sim 600 \mathrm{bp}$ fragment of leukolysin. To control for the amount of cDNA in each reaction, the same panels are amplified for the housekeeping gene GAPDH and presented at the lower panel.

\section{Proleukolysin is localized in secretory granules throughout the cell}

Since almost all intracellular leukolysin is in a latent state (Fig 3c), it must be residing in the trans-Golgi network waiting to be processed by furin or related proprotein convertases[12],[14]. To track the localization of proleukolysin, a GFP fusion construct was engineered to fuse leukolysin1-280 to the green flurorescent protein (GFP) (Fig 4a). Stable cell lines expressing the GFP fusion protein were generated from MDCK cells as described[19]. More than 15 positive clones were obtained and a few representatives are shown by zymographyin Fig $4 \mathrm{~b}$. As expected, GFP adds $22 \mathrm{kDa}$ to the secreted gelatinolytic speces as a $50 \mathrm{kDa}$ enzyme (Fig $4 \mathrm{~b}$ ). Clone 15-11 (lane 4 of Fig $4 \mathrm{~b}$ ) was selected for further analysis. Western blotting of the supernatants and cell lysates from 15-11 cells demonstrated that the majority of intracellular leukolysin:: GFP is still in latent form as demonstrated for leukolysin ${ }_{1-280}$ in Fig 3c (data not shown). To visualize the GFP tagged proleukolysin, confocal microscopy was performed to demonstrate that leukolysin $\sin _{1-280}:$ : GFP is localized in secretory granules throughout the cell body, contrary to the concentrated localization in perinuclear ER and Golgi network for most secretory proteins. Thus, the signal peptide and the prodomain of leukolysin contain the necessary information sufficient to target leukolysin to secretory granules, perhaps relevant to the ones found in leukocytes[4].

\section{DISCUSSION}

As a cell type critical to inflammatory response, leukocytes are very mobile, exiting the blood stream and entering the inflamed sites to destroy invading pathogens, infected host 

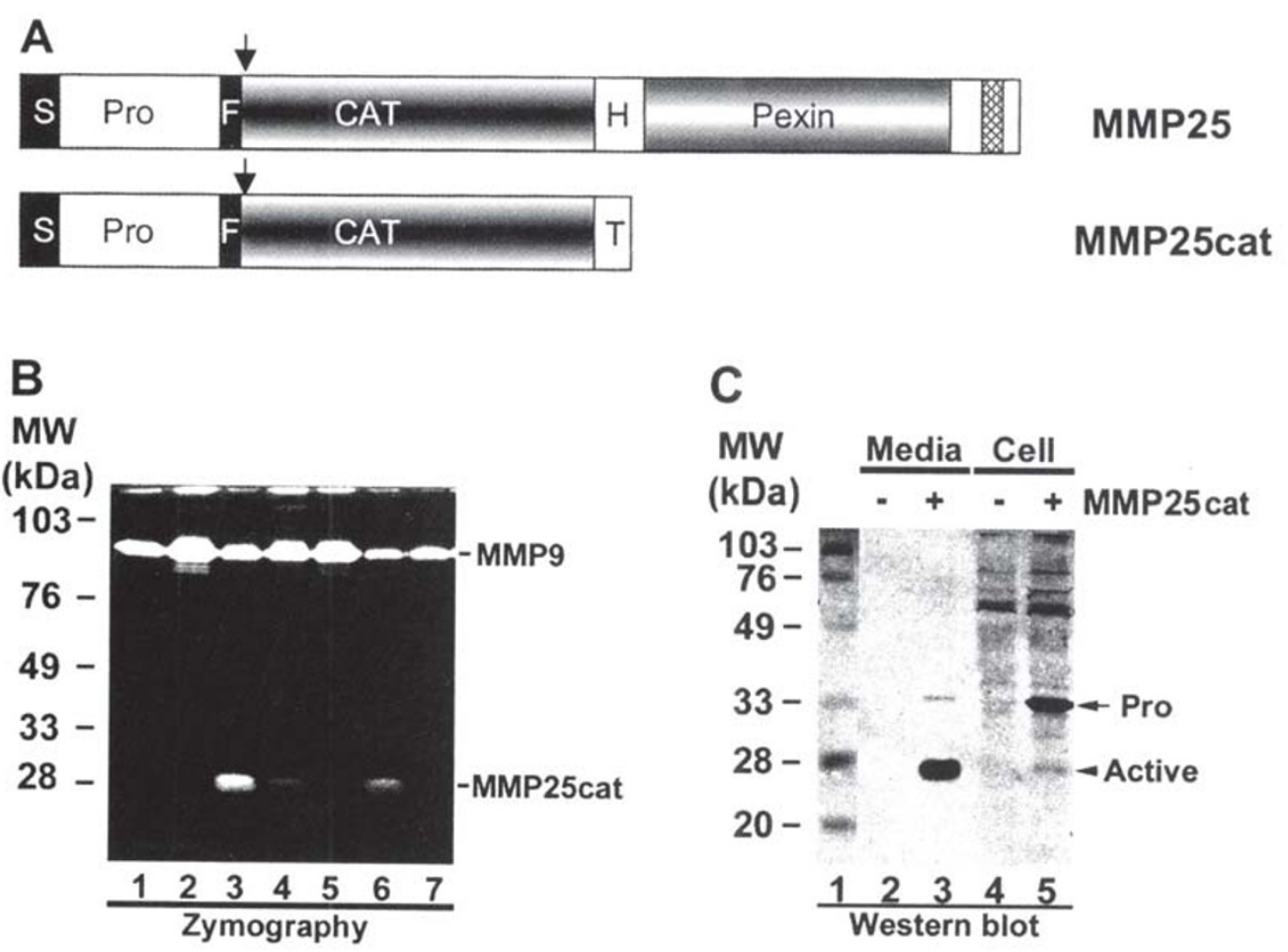

Fig 3.

Stable expression of leukolysin/MMP25 $5_{1-280}$.

A, Schematic diagram for the leukolysin expression construct. The upper portion indicates the full length leukolysin (MMP25) and the lower portion for leukolysin1-280 (MMP25cat) as a in- frame fusion with the FLAG tag as described in Materials and Methods. S: signal peptide; Pro: prodomain; F: furin; CAT: catalytic; H: hinge; Pexin: hemopexin-like; T: FLAG tag. B, A representative zymogram depicting a gelatinolytic band for leukolysin. Control (lane 1) and MMP25cat (lanes 2-7) transfected MDCK cells were stable clones and serum free supernatants from these clones were collected and analyzed by zymography as described[25]. Out of 6 clones shown, only three (lanes 3 , 4, and 6) are positive for leukolysin1-280. The high molecular weight gelatinolytic species is the endogenous MMP9 from MDCK cells. Clone \#17-13 was chosen for further study (lane 3). C, Analysis of intracellular and secreted leukolysin. Control (lanes 2 and 4) and MMP25cat17-13 (lanes 3 and 5) were grown to confluency and washed three times with PBS before replenished with serum free media. $48 \mathrm{~h}$ later, conditioned media were collected and the cells were lysed in RIPA buffer. The supernatants (lanes 1,2) and cell lysates (lanes 3,4) were analyzed by Western blotting using M2 antibody as described previously[9]. The pro- species is indicated by arrow and the active one by an arrowhead.

cells and damaged tissues[4]. Leukolysin fits quite naturally into the role of leukocytes as part of their proteolytic arsenals, perhaps complementing the roles of two MMPs previously found in leukocytes, neutrophils in particular: MMP8 and MMP9[26, 27]. The localization of a C-terminally truncated proleukolysin into the secretory granules of 
Molecular cloning of a novel MMP: MMP:25
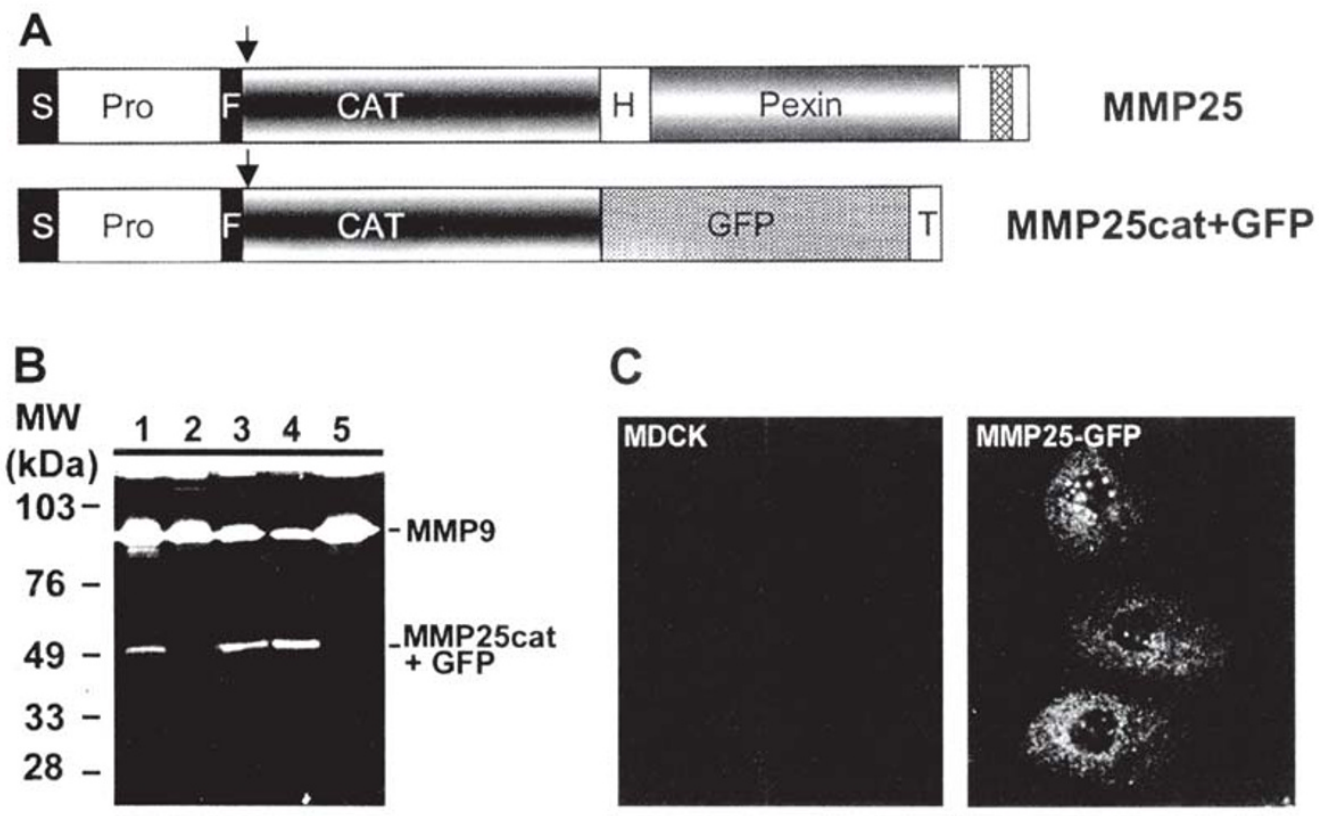

Fig 4.

Proleukolysin in secretory granules.

A, Schematic diagram of leukolysin-GFP construct. The structure of leukolysin is shown on the upper portion. Below is the in-frame fusion between leukolysin1-280 and GFP tagged with FLAG (T) (see Materials and Methods) designed to track the localization of proleukolysin insidethe cells. B, a representative zymogram of stable clones transfected with MMP25cat-GFP. Control (lane 5) and leukolysincat-GFP transfectants (lane 1-4) were grown to confluency and conditioned with serum free media which were subsequently analyzed as described in Fig 3. GFP contributes about $22 \mathrm{kDa}$ to the molecular weight of the fusion protein (50 vs $28 \mathrm{kDa}$, see Fig 3). C, Confocal localization of Leukolsyin/MMP25cat-GFP. The left panal is confocal image of control transfected cells from lane 5 of panel $\mathrm{B}$. The right panel is for cells transfected with MMP25cat-GFP (shown here is clone 15-11, lane 4 of panel B, but all clones behave identically) sectioned optically on a BioRad MRC confocal system. The intracellular proleukolysin is localized mainly in secretory granules throughout the cell bodies.

MDCK cells seems to suggest that leukolysin may be stored as zymogens in the intracellular granules of leukocytes-a depot of many powerful, yet latent, proteinases[4]. Although characterized as a potent proteinase against denatured collagen (Fig $3 \mathrm{~b}$ ), leukolysin's role in ECM destruction is not known at present. With the availability of purified enzymes and the full-length cDNA clone, the full potential of leukolysin as an ECM degrading enzyme is under investigation thoroughly. Furthermore, a genomic clone 
has been isolated from mouse SVJ129 genomic library (Yi and Pei, unpublished data). Given its tissue specific nature of expression, leukolysin deficient mice generated by genetic ablation should display functional deficiency in leukocyte- or inflammation related physiological as well as pathological processes such as inflammatory responses.

With a RRRR motif, leukolysin is apparently a new member of a MMP subgroup that can be activated by furin-type proprotein convertases[12],[24]. Although the precise mechanism for its activation remains to be investigated, proleukolysin ( $34 \mathrm{kDa})$ was shown to be processed intracellular into the $28 \mathrm{kDa}$ active species (Fig 3c), presumably by furin or related PCs. MMP activation mediated by furin or related proprotein convertases was first proposed during the characterization of stromelysin-3[12]. With the recent identification of leukolysin/MMP25, MT5-MMP/MMP24 and CA-MMP/MMP23, close to $40 \%$ of known MMPs ( 8 out of 20) possess a RXK/RR motif, ready to be cleaved and activated by furin or related PCs[5],[9],[11], [20],[23],[24]. Addition of leukolysin to this group further illustrates the importance of furin- or PC- mediated MMP activation in ECM remodeling processes.

By displaying the strongest homology to the MT-MMP subgroup, especially MT4-MMP (Fig 1c), leukolysin is also named MT6-MMP. Based on the alignment in Fig 1c, it seems plausible to divide the MT-MMPs into two branches: MT1, 2, 3, 5-MMPs and MT4, 6MMPs. The defining function for the former group is their ability to activate progelatinase $\mathrm{A}[15],[16],[20]$, whereas the later can not. Current efforts are underway to address the roles of signal peptide and prodomain in targeting proleukolysin to intracellular granules (Fig 4) and that of the C-terminal hydrophobic domain in cell membrane localization. Anchored on cell surface, active leukolysin could be in close contact with substrates and secluded from the anti-proteinase shield in the extracellular milieu, thus, may exert a unique force in destroying and remodeling extracellular matrix during leukocyte-mediated inflammatory responses.

\section{ACKNOWLEDGEMENT}

The author wishes to thank members of the Pei laboratory for a stimulating environment and skillful assistances; Drs. Hideaki Nagase, Fred Woessner and Stephen J. Weiss for thoughtful inputs and comments for this study. This study was supported in part by grant CA76308 from the National Cancer Institute, Elsa Pardee Foundation, University of Minnesota grant-in-aid, Minnesota Medical Foundation.

\section{REFERENCES}

[1] Woessner JF Jr. Matrix metalloproteinases and their inhibitors in connective tissue remodeling. Faseb J 1991; 5:2145-54.

[2] Hahn-Dantona E, N Ramos-DeSimone, J Sipley, H Nagase, DL French, JP Quigley. Activation of proMMP-9 by a plasmin/MMP-3 cascade in a tumor cell model. Regulation by tissue inhibitors of metalloproteinases. Ann N Y Acad Sci 1999; 878:372-87.

[3] Woolley DE, ED Harris, Jr CL Mainardi, CE Brinckerhoff. Collagenase immunolocalization in cultures of rheumatoid synovial cells. Science 1978; 200:773-5. 


\section{Molecular cloning of a novel MMP: MMP:25}

[4] Weiss SJ. Tissue destruction by neutrophils [see comments]. N Engl J Med 1989; 320:365-76.

[5] Massova I, LP Kotra, R Fridman, S Mobashery. Matrix metalloproteinases: structures, evolution, and diversification. Faseb J 1998; 12:1075-95.

[6] Stocker W, F Grams, U Baumann, P Reinemer, FX Gomis-Ruth, DB McKay, W Bode. The metzincinstopological and sequential relations between the astacins, adamalysins, serralysins, and matrixins (collagenases) define a superfamily of zinc-peptidases. Protein Sci 1995; 4:823-40.

[7] Nagase H, JF Woessner, Jr. Matrix metalloproteinases. J Biol Chem 1999; 274:21491-4.

[8] Van Wart HE, H Birkedal-Hansen. The cysteine switch: a principle of regulation of metalloproteinase activity with potential applicability to the entire matrix metalloproteinase gene family. Proc Natl Acad Sci USA 1999; 87:5578-82.

[9] Pei D. CA-MMP: a matrix metalloproteinase with a novel cysteine array, but without the classic cysteine switch(1). FEBS Lett1999; 457:262-70.

[10] Muller D, B Quantin, MC Gesnel, R Millon-Collard, J Abecassis, R Breathnach. The collagenase gene family in humans consists of at least four members. Biochem J 1988; 253:187-92.

[11] Velasco G, AM Pendas, A Fueyo, V Knauper, G Murphy, C Lopez-Otin. Cloning and characterization of human MMP-23, a new matrix metalloproteinase predominantly expressed in reproductive tissues and lacking conserved domains in other family members. J Biol Chem 1999; 274:4570-6.

[12] Pei D, SJ Weiss. Furin-dependent intracellular activation of the human stromelysin-3 zymogen. Nature 1995; 375:244-7.

[13] Santavicca M, A Noel, H Angliker, I Stoll, JP Segain, P Anglard, M Chretien, N Seidah, P Basset. Characterization of structural determinants and molecular mechanisms involved in pro- stromelysin3 activation by 4-aminophenylmercuric acetate and furin-type convertases. Biochem J 1996; 315:9538.

[14] Steiner DF. The proprotein convertases. Curr Opin Chem Biol 1998; 2:31-9.

[15] Sato H, T Takino, Y Okada, J Cao, A Shinagawa, E Yamamoto, M Seiki. A matrix metalloproteinase expressed on the surface of invasive tumour cells [see comments]. Nature 1994; 370:61-5.

[16] Takino T, H Sato, A Shinagawa, M Seiki. Identification of the second membrane-type matrix metalloproteinase (MT-MMP-2) gene from a human placenta cDNA library. MT-MMPs form a unique membrane-type subclass in the MMP family. J Biol Chem 1995; 270:23013-20.

[17] Will H, B Hinzmann. cDNA sequence and mRNA tissue distribution of a novel human matrix metalloproteinase with a potential transmembrane segment. Eur J Biochem 1995; 231:602-8.

[18] Puente XS, AM Pendas, E Llano, G Velasco, C Lopez-Otin. Molecular cloning of a novel membranetype matrix metalloproteinase from a human breast carcinoma. Cancer Res 1996; 56:944-9.

[19] Pei D, J Yi. A high-level mammalian expression system based on the Madin-Darby canine kidney cell line. Protein Expr Purif 1998; 13:277-81.

[20] Pei D. Identification and characterization of the fifth membrane-type matrix metalloproteinase MT5MMP [In Process Citation]. J Biol Chem 1999; 274:8925-32.

[21] Lennon G, C Auffray, M Polymeropoulos, MB Soares. The I.M.A.G.E. Consortium: an integrated molecular analysis of genomes and their expression. Genomics 1996; 33:151-2.

[22] Kajita M, H Kinoh, N Ito, A Takamura, Y Itoh, A Okada, H Sato, M Seiki. Human membrane type4 matrix metalloproteinase (MT4-MMP) is encoded by a novel major transcript: isolation of complementary DNA clones for human and mouse mt4-mmp transcripts. FEBS Lett 1999; 457:353-6.

[23] Llano E, AM Pendas, JP Freije, A Nakano, V Knauper, G Murphy, C Lopez-Otin. Identification and characterization of human MT5-MMP, a new membrane-bound activator of progelatinase a overexpressed in brain tumors. Cancer Res 1999; 59:2570-6.

[24] Nagase H. Activation mechanisms of matrix metalloproteinases. Biol Chem 1997; 378:151-60.

[25] Pei D, SJ Weiss. Transmembrane-deletion mutants of the membrane-type matrix metalloproteinase1 process progelatinase A and express intrinsic matrix-degrading activity. J Biol Chem 1996; 271:913540. 
[26] Hasty KA, TF Pourmotabbed, GI Goldberg, JP Thompson, DG Spinella, RM Stevens, CL Mainardi. Human neutrophil collagenase. A distinct gene product with homology to other matrix metalloproteinases. J Biol Chem 1990; 265:11421-4.

[27] Hibbs MS, KA Hasty, JM Seyer, AH Kang, CL Mainardi. Biochemical and immunological characterization of the secreted forms of human neutrophil gelatinase. J Biol Chem 1985; 260:2493-500.

Received Nov.10,1999

Accepted Nov.26,1999 